\title{
Germanica
}

\section{La marche à la mort : Vienne et Prague crépusculaires chez Hofmannsthal et Leppin}

Todessehnsucht. Wiener und Prager Endzeitstimmung bei Hofmannsthal und Leppin

\section{Patrick Bergeron}

\section{OpenEdition}

Journals

Édition électronique

URL : http://journals.openedition.org/germanica/551

DOI : 10.4000/germanica.551

ISSN : 2107-0784

\section{Éditeur}

Université de Lille

\section{Édition imprimée}

Date de publication : 1 décembre 2008

Pagination : 41-51

ISBN : 978-2-913857-22-6

ISSN : 0984-2632

\section{Référence électronique}

Patrick Bergeron, «La marche à la mort : Vienne et Prague crépusculaires chez Hofmannsthal et Leppin », Germanica [En ligne], 43 | 2008, mis en ligne le 01 décembre 2010, consulté le 06 octobre 2020. URL : http://journals.openedition.org/germanica/551 ; DOI : https://doi.org/10.4000/germanica. 551

Ce document a été généré automatiquement le 6 octobre 2020.

(c) Tous droits réservés 


\title{
La marche à la mort : Vienne et Prague crépusculaires chez Hofmannsthal et Leppin
}

Todessehnsucht. Wiener und Prager Endzeitstimmung bei Hofmannsthal und Leppin

\author{
Patrick Bergeron
}

1 Hugo von Hofmannsthal (1874-1929) et Paul Leppin (1878-1945) ont tous deux invalidé l'esprit de provincialisme imprégnant leurs villes respectives, Vienne et Prague, au moment où Berlin commençait à s'imposer comme capitale germanophone de la modernité. Hofmannsthal a fait fructifier l'héritage millénaire et universel des Habsbourg, et assumé au grand jour les composantes germanique, romane et slave de ce legs. Leppin a enregistré les prestiges troublants du vieux Prague, quitte à se perdre dans l'abîme. Chez les deux écrivains s'est énoncée une expérience esthétique marquée par des valeurs crépusculaires (mélancolie, errance, haine de soi, complaisance dans le macabre, parmi d'autres) où triomphait un esprit de modernité original, puisque dégagé du naturalisme rayonnant à Berlin mais que Hermann Bahr invitait, dès 1891, à dépasser. Cet esprit de modernité est original, de surcroît, puisqu'il s'appuie sur la réappropriation, plutôt que le rejet, des valeurs esthétiques du passé. L'étude qui suit se propose de situer la sensibilité crépusculaire émanant de la «Jeune Vienne»par rapport à celle de la "Jeune Prague », telles que représentées par Hofmannsthal et Leppin.

\section{Vienne moderne. Le moi se meurt ${ }^{1}$}

2 Il n'est pas souvent question de Vienne directement dans l'œuvre de Hofmannsthal, celui-ci ne décrivant pas, ou fort peu, la cité impériale. Pourtant, son œuvre ne cesse d'affirmer l'esprit viennois, un esprit reconnaissable à la vigueur et l'étendue des racines culturelles. L'Espagne baroque, le Paris des comédies-ballets, la Venise des 
Lumières, l'Orient des Mille et Une Nuits... L'œuvre de Hofmannsthal s'appuie sur la conviction du monde reçu en héritage.

3 Ce monde, c'est aussi celui des contemporains. Hofmannsthal était encore lycéen lorsqu'il a commencé à rendre compte d'ouvrages récents pour différentes revues et journaux de langue allemande. Les textes recensés composent à eux seuls un florilège de littérature actuelle : Physiologie de l'amour moderne (1890) de Paul Bourget, le drame Die Mutter (1891) de Hermann Bahr, les trois volets du Culte du Moi (1888-1891) de Maurice Barrès, le Fragment d'un journal intime (1883) de Henri-Frédéric Amiel. À cela s'ajoutent des articles sur Swinburne, Pater, Wilde, Ibsen, Marie Bashkirtseff, D'Annunzio, de même que sur les œuvres de compatriotes, tels Ferdinand von Saar, Peter Altenberg ou Arthur Schnitzler. Considérés d'un bloc, les premiers essais de Hofmannsthal s'ordonnent autour du phénomène de la quête du moi, qui se vit par extension comme une recherche d'originalité, de grandiose, de sens et d'unité, mais aussi comme la poursuite d'une forme devant compenser l'absence de style de l'époque moderne en comparaison des âges d'or du passé. En ressort une littérature concentrée sur le soi qui tend, donc, à se désolidariser d'autrui et exalter ou nier l'existence, les séductions de Schopenhauer et de Nietzsche étant quasi concomitantes dans ce contexte.

4 Ce double élan d'individualisme et de pessimisme correspond à ce que Hofmannsthal appelle, dans un essai de 1892 sur Schloß Kostenitz de F. von Saar, " une atmosphère autrichienne fondamentale: intérieure, aux sentiments délicats et marquée par la crainte de la vie ${ }^{2}$ ». Les personnages de Saar ont « presque tous » tendance à fuir la vie. Mais « "fuir" n'est pas le bon terme », explique Hofmannsthal :

[s]ans violence et sans pathos accusateur, c'est une sortie discrète et timide, comme on quitte une compagnie énervante et pénible. Il y a chez la plupart quelque chose de désemparé et de féminin ; ils oublient volontiers et facilement le commerce des autres hommes ; ils aiment s'entourer de vieilles choses fanées et très usées ; ce qui est étranger à la réalité leur fait du bien et ils sont très fortement soumis à la fascination mystérieuse du passé ${ }^{3}$.

5 Cet art mélancolique et baroque de la sortie de scène après que chacun eut joué son rôle se retrouve également chez Schnitzler. Dans Ein Abschied (« Un adieu », 1895), bref récit dont l'un des titres provisoires a été Der Wartende ("Celui qui attend»), Schnitzler raconte les heures d'attente inquiète et confuse d'Albert, qui découvre progressivement pourquoi sa maîtresse, Anna, ne s'est pas présentée à leur dernier rendez-vous clandestin : elle vient de succomber à une encéphalite typhique. Le sort de la défunte est ici prétexte à un tournoiement de pensées macabres et saugrenues chez « celui qui attend». Ce que Schnitzler décrit, c'est l'attente d'une compréhension: l'événement principal de la nouvelle n'est pas tant la mort de la jeune fille que l'enchaînement d'impressions dans l'esprit du jeune homme (comme c'est également le cas dans La mort de Georges de Richard Beer-Hofmann). Le personnage se met à comprendre la réalité de la mort comme perte de contact avec la vie et plongée hors du temps.

6 Dans ses drames lyriques, Hofmannsthal mettra lui aussi en scène des personnages qui sont les spectateurs de leur propre vie (Andrea, Claudio, les élèves du Titien). Cette caractéristique traduit une impression qu'il retrouve chez ses contemporains. Rappelons les lignes que lui a inspirées le cycle dramatique Anatol en 1892 :

Aussi jouons-nous du théâtre.

Nous jouons nos propres pièces,

Mûris précocement, tendres et tristes, 
Nous jouons les comédies de notre âme

[...] Des agonies, des épisodes ${ }^{4}$...

7 Les agonies en question sont fictives ; l'existence se compose d'aventures épisodiques, déliées les unes des autres. L'individu, comme le dépeint Schnitzler dans Anatol, croit trouver son unité dans la disparité, l'excentricité des humeurs, l'agencement d'éléments flottants et de joies volatiles - un scénario que reprendra à son compte Leppin en montrant Severin et Blaugast se remémorant les épisodes déliés de leur vie sentimentale -, mais son mode de vie confine à la solitude, à l'irréalité, à la mélancolie et au harcèlement de la conscience. Esthétiser la vie, c'est la théâtraliser. La théâtraliser, c'est la dépouiller d'émotions authentiques et la remplir de constructions de l'esprit.

Le schönes Leben de l'esthète, ce mode de vie qui produit des individualités supérieurement raffinées, en fait aussi des êtres supérieurement démunis parce que privés des liens authentiques sans lesquels l'expérience de l'unité est impossible. Andrea, héros du drame Gestern, les élèves du Titien dans Der Tod des Tizian et Claudio, dans Der Tor und der Tod, auront finalement tort de se réfugier dans un univers de beautés comme dans un nid d'aigle, au-dessus ou loin du monde. Leur existence est unidimensionnelle. Ce sont des spectateurs. Il leur manque la dimension complémentaire à la sensibilité artiste, l'éthique, qui les humaniserait.

Nulle conquête ne paraît possible sans une réaffectation des composantes de la recherche : le moi, à la poursuite de lui-même, finit par se voir fragilisé, en quête d'un sens (ou d'un centre). La voie du moi mène vers la révélation de ses défaillances, de son instabilité, de son indécision, de sa vulnérabilité, de ses contradictions - ou, chez Leppin, de sa négation.

10 La revendication individualiste aboutit ainsi à la découverte d'une disparité qui fait défaillir, qui affole et rend redoutable la perspective de laisser échapper la vie. À propos d'un roman du Norvégien Arne Garborg (1851-1924), Âmes lasses (1890), l'essayiste viennoise Marie Herzfeld observait :

C'est pourquoi les forces de notre âme ne convergent pas vers un point de direction. Elles n'obéissent pas à une impulsion centrale, mais se retournent les unes contre les autres. Elles se paralysent et se détruisent mutuellement dans un scepticisme dévorant. Elles nous rendent inaptes à la volonté patiente, au sentiment fort, à l'existence joyeuse et à la mort courageuse ${ }^{5}$.

11 La disparité qui fait défaillir continuera d'occuper une position privilégiée dans l'œuvre de Hofmannsthal postérieure à 1900. De La lettre de Lord Chandos au fragment d'Andreas, le moi est fragilisé, à la dérive, soumis à une inquiétante labilité. L'unité, dont l'absence le fait défaillir, se situe peut-être, en définitive, du côté de la mort. "Tes pas te portent là où tu dois mourir ${ }^{6} "$, écrivait Hofmannsthal dans Le Conte de la $672^{e}$ nuit (1895).

12 La mort gronde ou guette, prête à survenir à tout moment et en tout lieu. Maeterlinck a fait d'elle le thème central de sa série de pièces L'Intruse (1890), Les Aveugles (1890), Les Sept Princesses (1891), Intérieur (1894), La Mort de Tintagiles (1894), parues en version allemande dès 1901. Maeterlinck a fourni l'inspiration à Hofmannsthal pour créer des atmosphères d'une étrange indétermination. Dans «La scène, image de rêve » (1903), Hofmannsthal mentionne «les terribles murailles de mort, où les créatures de Maeterlinck s'ensanglantent les mains à frapper contre les blocs de pierre de leur base avant qu'elles se consument sans qu'on les entende ${ }^{7}$ ». L'inspiration maeterlinckienne paraît évidente dans le drame Das Bergwerk zu Falun, ainsi que dans les récits Das 
Märchen der 672. Nacht et Reitergeschichte, alors qu'un destin incompréhensible et apparemment incontrôlable est à l'œuvre qui pousse les personnages vers une mort mystérieuse.

L'exemple du fils du marchand, dans Le Conte de la $672^{\circ}$ nuit, est éloquent à cet égard. Le héros, jeune, riche et solitaire, vit dans un univers saturé de raffinement et de grâce, et il est lui-même doté d'un physique harmonieux. Mais il éprouve un sentiment de manque, de vide, d'inassouvissement qui l'incite à tout quitter, sous prétexte d'une affaire de courrier incriminant à élucider. Il part, en réalité, s'initier à la force impérieuse de la vie, mais aussi à la rencontre d'un destin implacable et angoissant, car la mort l'attend au bout du chemin, avec le sentiment désolant d'avoir raté sa vie.

Une situation analogue s'observe dans Histoire de cavalerie. En juillet 1848, pendant la campagne milanaise de l'armée autrichienne, le maréchal des logis Anton Lerch se laisse entraîner dans une rêverie vertigineuse et dans la traversée énigmatique d'un village déserté où surgissent diverses apparitions. À la fin de la nouvelle, il s'insurge muettement contre le capitaine baron Rofrano, qui donne l'ordre de libérer les chevaux de main capturés ; son immobilité lui vaudra de finir fusillé. Une force obscure, tapie dans les profondeurs ignorées de Lerch, l'avait fixé dans un inexplicable refus. Il s'agit, à nouveau, d'une marche à la mort.

\section{Prestiges troublants du vieux Prague}

15 Alors qu'il est peu question de la ville de Vienne elle-même dans l'œuvre de Hofmannsthal, il en est tout autrement de Prague dans l'œuvre, moins abondante, du poète, dramaturge et romancier tchèque Paul Leppin. On découvre vite, en effet, l'omniprésence de la ville dorée (surtout le Prague nocturne) chez l'auteur de Daniel Jesus, en particulier dans ses romans Marche dans les ténèbres et Au-dessus de tout. Le sous-titre de Marche dans les ténèbres, "Un roman des fantômes de Prague ", traduit bien le choix de Leppin de considérer Prague comme un univers nimbé d'irréalité.

Le Prague de Leppin est proche de celui de Meyrink : haut lieu du fantastique occulte, il s'écarte de la réalité ordinaire. Comme chez Hofmannsthal, l'espace où évoluent les personnages est un lieu d'égarement, de perdition. Mais le Prague de Leppin appelle, surtout, bon nombre de rapprochements avec l'art français et belge du portrait de ville en période symboliste, ce qui fait de Paul Leppin un proche parent de Huysmans, de Rodenbach, de même qu'un héritier de Baudelaire. Max Brod, qui l'a côtoyé, est de cet avis dans son Autobiographie (1964):

Leppin avait été littéralement élu pour chanter le vieux Prague qui s'éteignait alors dans les douleurs, avec ses ruelles mal famées, ses nuits de beuverie, ses vagabonds, ses saints pompeusement baroques à la religiosité suspecte... [...] Il fut une sorte de Baudelaire germano-tchèque. Mais privé de tout espoir de rédemption ${ }^{8}$.

17 Brod a raison de mentionner cette privation de "tout espoir de rédemption » : si la marche à la mort, chez Hofmannsthal, connaissait un dénouement sinon heureux, du moins ouvert à la transfiguration, quand l'éthique suppléait à l'esthétique, elle devient plongée vertigineuse dans la nuit et la déchéance chez Leppin. L'auteur donne vie à un Prague canaille, composé de grisettes musiciennes, d'anarchistes poseurs de bombes, d'ivrognes libidineux et de poètes loqueteux. Cet envers glauque de la Belle Époque permet à Leppin de projeter ses protagonistes Severin et Blaugast aux tréfonds de l'abjection et de la haine de soi. Leur chute paraît effrénée ; tout le vieux Prague, à la 
ronde, leur en offre non pas l'avertissement, car l'éthique n'a guère sa place ici, mais le constant spectacle. Or, comme l'a observé Bernard Michel, ce n'est pas Prague qui provoque la perte des héros de Leppin, car le mal se trouve à l'intérieur d'eux, «tapi dans la nature humaine »; leur pire ennemi est eux-mêmes, car finalement « [leurs] promenades dans Prague et ses faubourgs sont des moments de fraîcheur et de bonheur" ». Par la splendeur de son délabrement et son appel aux sens dans un tourbillon de forces vitales où la mort n'est jamais absente, le Prague de Leppin s'impose, à l'instar de la Vienne hofmannsthalienne (mais avec un surcroît de morbidité), comme une capitale mitteleuropéenne de la culture des sentiments et du délabrement exquis.

Marche dans les ténèbres (1914) est avant tout rythmé par les entrées et les sorties de Severin, ainsi que par le cycle des saisons, avec les impressions que l'été finissant ou l'hiver débutant déposent dans l'âme de Severin. C'est l'imprégnation de l'atmosphère qui donne à Prague, au fil des pages, un relief singulier et subjectif, dans un esprit proche des notations dont Peter Altenberg a fait sa spécialité : l'atmosphère pragoise qu'enregistre Leppin est nostalgique, lugubre et vague ; l'aujourd'hui est grignoté par l'autrefois, et la réalité se poursuit dans ses marges. Au gré des déambulations nocturnes de Severin dans Prague, le constant rappel de la mort (des suicides surviennent ou alimentent la rumeur, une jeune noyée est repêchée dans la Moldau, Severin a des envies de meurtre...) permet le développement d'un synopsis récurrent dans les narrations décadentes : l'accès à une vraie perception de la vie multiforme se fait sur fond d'errance, de folie et de mort. Et encore: il s'agit d'art suggestif et d'existences ratées. La vie, le destin échappent aux protagonistes de Leppin.

Les liens sont directs, dès lors, entre le décadentisme de Leppin et celui du jeune Hofmannsthal : on observe un même effacement des actions au profit des sensations, ces dernières ayant le poids d'événements vécus, car le ressenti (y compris le souvenir des impressions anciennes) l'emporte sur l'agir. Le personnage est porté davantage qu'il ne se transporte; les choses lui arrivent, son détachement (surtout sentimental) est une constante; des forces inconnues, invisibles, agissent dans l'ombre. Un autre aspect appelle le rapprochement avec Hofmannsthal : les efforts que fait l'individu pour maitriser ou comprendre ses désirs, ont souvent pour issue le fléchissement de sa volonté.

À 23 ans, Severin passe ses matinées à étouffer dans un bureau affreux, ses après-midis à dormir sur son canapé de cuir noir, et n'en sort qu'après qu'on eut allumé les réverbères. Il vagabonde alors dans les gargotes et les cafés ouverts la nuit. Ses promenades nocturnes sont l'affirmation de son attente inquiète et trouble. Il sent l'approche d'un destin inconnu, s'en effare parfois, s'en moque d'autres fois. D'humeurs volatiles, il sentira, l'été, qu'il tourne en rond comme un animal enchaîné à un piquet. L'hiver revenu, il aura l'impression que quelque chose de nouveau se produit, quelque chose qui lui manquait depuis longtemps. Il n'a pas de prise rationnelle (et rassurante) sur le contenu de son existence. D'où, sans doute, sa tendance à remonter le cours du temps de sa vie personnelle, souvent jusqu'à ses souvenirs d'enfance. D'où, également, son attitude de spectateur de lui-même, lorsque le surprend une sensation confuse: «Sa conscience se dissociait et vivait, séparée, sa propre vie. Passé et présent défilaient devant lui comme les images d'un paysage et il regardait, étonné et passif, sa propre existence $^{10} »$. 
21 Pour l'essentiel, cette existence se compose d'aventures érotiques et de rencontres aléatoires. Prague, ville aux murs sombres et au délabrement doré, est trop thématisé pour n'être qu'un simple décor. Severin connaît par cœur son «langage silencieux »; « entre ses murs noircis, ses tours et ses demeures nobles, au contact de son étrangeté sans vie, tout un univers fantasmagorique inexprimé avait grandi avec lui, et [il] ne pouvait mettre le pied dans la rue sans avoir l'impression que son destin l'attendait ${ }^{11}$ ". La ville possède ainsi un côté cabalistique ; elle n'agit que sur les initiés, et Nathan Meyer - ce « chasseur d'âmes » qui, comme Shobotzki dans Au-dessus de tout, se repaît des malheurs d'autrui, a tôt reconnu Severin comme un membre de la " guilde » : « Il y avait des êtres pour qui l'éclat du monde n'était qu'illusion. Des cyniques infortunés, des parias qu'une peur animale chassait dans les rues, des assassins et des stigmatisés. Telle était la guilde à laquelle Severin appartenait, lui aussi ${ }^{12}$ ».

Pour Severin, l'enchantement se produit avant l'aube. De retour de ses errances nocturnes, " [s]es sens étaient vifs et alertes; il voyait la nuit transformer les choses, leur donnant une seconde vie, différente de leur vie diurne. Il voyait comme elle changeait les places nues et désertes en paysages mélancoliques, les ruelles étroites en caveaux aux murs humides ${ }^{13} »$. Dans cette magie nocturne, la mort revêt un prestige incomparable: «La mort. Dans la sonorité sourde de cette syllabe, il y avait quelque chose qui lui paraissait plus excitant, plus riche de sens que toutes les manifestations somnolentes d'une vie protégée ${ }^{14}{ }^{\prime}$.

Cette référence à Thanatos, support à des images pharmaceutiques (la mort comme excitant) et artistiques (la mort riche comme une belle œuvre d'art), s'éclaire par le contexte des impressionnistes et décadents de France et de Belgique, que les auteurs du Jeune Prague lisaient en traductions dans la Moderni revue. Qu'on pense au drame de Rachilde, Madame la Mort, à Bruges-la-Morte et Le Carillonneur de Rodenbach ou au petit traité de délectation morose qu'est Du sang, de la volupté et de la mort de Maurice Barrès : on comprend la tradition décadente qui précède Leppin dans l'énoncé de ce fantasme de domination individualiste de la mort : "Voilà la mort ", s'exclame Severin lorsqu'il tend à son amie Suzanne un paquet de viande empoisonnée (qui sera fatale à son animal de compagnie, Anton, un corbeau). Comme l'a expliqué Jules Laforgue : «rien n'est plus chatouilleux aux organismes supérieurs que [de] se sentir mourir tout en sachant qu'il n'en sera rien, le crépuscule et l'automne, le drame du soleil et de la mort sont esthétiques par excellence ${ }^{15}$ ». Marche dans les ténèbres s'inscrit dans cette " pompe funèbre ".

Dans un ordre de préoccupations identiques, Au-dessus de tout (achevé vers 1932, publié posthumément en 1984) est l'histoire d'un dépérissement. Klaudius Blaugast, un petit employé blasé, poursuit comme Severin la pente de ses désirs. Dans son cas, le destin inconnu va le propulser dans un tourbillon de dégradation. Il se laisse d'abord dévoyer par Shobotzki, un ancien camarade de classe, qui propose de l'initier à une science de la dégradation. Déclassé et démuni, Blaugast perd tout à cause de la cruelle prostituée Wanda, qui en fait son esclave sexuel, puis son homme à tout faire. Il cherche le salut dans un Prague festif et licencieux, mais qui demeure insensible à son malheur. Exclu parmi les exclus, Blaugast s'enfonce dans la honte et l'abjection : après avoir été au service de la prostituée Wanda, il devient un mendiant à qui les clients des bouges demandent d'imiter le cri de l'oiseau et que de riches débauchés payent pour voir déverser sa semence dans une assiette en écoutant ses râles. Son seul soutien lui vient de Johanna, une prostituée dont la mère est morte de syphilis, qui entreprend de le 
sauver. Mais aucun salut n'est possible ici, Max Brod avait vu juste; au mieux, le personnage peut-il espérer de trouver un abri ou refuge. Occupant le lit de la défunte «maminka », il a vaincu le grondement de la rue, grâce à Johanna, qui a privé celle-ci de son pouvoir funeste.

Différentes caractéristiques de Marche dans les ténèbres reviennent dans Au-dessus de tout : la construction du récit autour d'un protagoniste errant, les interruptions du récit par des retours en arrière et la remémoration des épisodes de sa vie sentimentale, la prédominance d'un Prague nocturne et canaille, parmi d'autres. Or Leppin a ajouté une dimension outrancière qui éclaire sous un autre jour la teneur désespérée de Marche dans les ténèbres. Ainsi Blaugast, amant inconstant et frivole, vit des "épisodes" sentimentaux autrement plus déroutants que Severin. Celui-ci dominait ses aventures; alors que pour Blaugast, elles sont un assemblable d'histoires putrides à l'origine sinon du mal, du moins de la honte qu'il porte en lui et qui décide de sa destinée : « Le mal est en moi. Ma perception du monde a subi une transformation chimique, toujours elle suit, étouffante, le trajet tortueux de ce nerf, toujours le même chemin, du cerveau jusqu'aux reins ${ }^{16} »$.

Le fantasmagorique prend plus de place ici que dans Marche dans les ténèbres. Par exemple, un chapitre entier est consacré à l'hallucination d'une jungle en rut. Leppin se livre à une exploration des tendances sadiques enfouies en chacun. Son Blaugast est un Severin vieilli. Il se souvient de sa jeunesse, vingt ans plus tôt, et son histoire montre les ravages que l'inanité a laissés sur son existence :

Cette terre était le grand bazar du mal, qui tirait sournoisement sur sa chaîne. Ses espions étaient partout. Aux coins venteux des rues, où des fillettes au visage fané vendaient des fleurs et des allumettes, sur la table d'opération des cliniques, dans les taudis des faubourgs, dans les gares et sous les ponts. La pitié n'était que le masque de la volupté, la bonté celui de la convoitise ${ }^{17}$.

Fait exception à ce tableau dégradé : la prostituée charitable Johanna, pour qui la volupté est le masque de la pitié. C'est elle qui porte secours à Blaugast après qu'un assaut de Shobotzki l'eut laissé brisé. Dans cet univers impitoyable au «baronnet en guenilles majestueuses » qu'est devenu Blaugast, Leppin repousse constamment les limites de la négation de soi. Brave " fille ", Johanna est la seule à vouloir protéger celui qu'elle nomme "mon frère malade ». C'est toujours la marche à la mort, mais le marcheur n'est plus seul.

\section{Marches funèbres}

Mélancolie, errance, haine de soi, complaisance dans le macabre... Ces valeurs crépusculaires occupent l'avant-plan des premiers poèmes, drames et récits de Hofmannsthal, de même que des romans de Leppin. L'absence à la vie, le cycle «vie (passive) / analyse du vécu ", la fascination de l'inaboutissement, la crainte de l'affadissement: voilà autant d'éléments qui justifient le rapprochement des deux auteurs et situent leur inspiration dans un cadre qui doit beaucoup à l'impressionnisme français et belge. Ce contexte a vu naître Bruges-la-Morte (1892), le roman de Georges Rodenbach qui porte sur le thème du deuil exagéré. Le protagoniste Hugues Viane fait son apparition sur fond de jour déclinant. Inoccupé, solitaire, il passe la journée enfermé dans sa chambre, se répétant avec un accent nervalien : " "Veuf! Être veuf ! Je suis le veuf!" Mot irrémédiable et bref! d'une seule syllabe, sans écho. Mot impair et 
qui désigne bien l'être dépareillé18 ${ }^{18}$. Vienne et Prague crépusculaires, tels que représentés par Hofmannsthal et Leppin, atteignent le faîte de leur modernité dans leur désignation de l'être dépareillé et des ratés de son existence.

\section{NOTES}

1. Cette partie s'appuie sur une analyse effectuée dans P. Bergeron, Aspects de la mort chez Maurice Barrès et Hugo von Hofmannsthal, thèse, Université Paul-Valéry Montpellier 3 et Université Laval, 2004.

2. H. v. Hofmannsthal, «Ferdinand von Saar : Le Château de Kostenitz » dans F. Saar, Le Château de Kostenitz, traduit par J.-F. Boutout, Paris, Le Promeneur, « Le Cabinet des lettrés », 2003, p. 10.

3. Ibid., p. 10-11.

4. Id., "Prologue à l'Anatale d'Arthur Schnitzler », dans Le lien d'ombre, traduit par J.-Y. Masson, Paris, Verdier, « Verdier poche », 2006, p. 125-127.

5. M. Herzfeld, «Fin-de-Siècle» (1892), version abrégée reproduite dans G. Wunberg (dir.), Die Wiener Moderne, Stuttgart, Reclam, 2000, p. 260-261 (nous traduisons). Die Kräfte unserer Seele wirken daher nicht gemeinsam Einem Richtungspunkte zu; sie gehorchen nicht einem zentralen Impulse, sondern kehren sich widereinander; sie lähmen und zerstören sich gegenseitig in fressender Skepsis; sie machen uns unfähig zu geduldigem Wollen, zu starkem Fühlen, zu freudigem Dasein und zu mutigem Tod.

6. H. v. Hofmannsthal, Andreas et autres récits, traduit par E. Badoux et M. Michel, Paris, Gallimard, « L'Imaginaire », 1985, p. 105.

7. H. v. Hofmannsthal, La Lettre de Lord Chandos et autres essais, traduit par A. Kohn et J.-

C. Schneider, Paris, Gallimard, « Du monde entier »,1980, p. 99.

8. Max Brod, Autobiographie, cité dans P. Leppin, Marche dans les ténèbres, traduit par C. Gepner, Paris, Phébus, 2001, p. 11-12.

9. B. Michel, Prague, Belle Époque, Paris, Aubier, « Collection historique », 2008, p. 135.

10. P. Leppin, Marche dans les ténèbres, p. 148.

11. Ibid., p. 39.

12. Ibid., p. 143.

13. Ibid., p. 68.

14. Ibid., p. 54.

15. J. Laforgue, «Persée et Andromède », dans Moralités légendaires, Paris, P.O.L., " La Collection », 1992, p. 133.

16. P. Leppin, Au-dessus de tout, traduit par C. Gepner, Paris, Phébus, 2004, p. 56.

17. Ibid., p. 30-31.

18. G. Rodenbach, Bruges-la-Morte, Arles, Actes Sud, Bruxelles, Labor, « Babel », 1989, p. 19. 


\section{RÉSUMÉS}

Figures de proue de la Jeune Vienne et de la Jeune Prague, Hugo von Hofmannsthal et Paul Leppin ont énoncé, à la veille de 1900, une modernité différente, par son attachement au passé, de celle rayonnant à la même époque à Berlin. Alors que le Viennois n'avait de cesse d'affirmer (et d'affermir) ses liens avec les cultures romane, germanique et slave, toutes trois constitutives de son identité, et que le Pragois capturait les prestiges troublants du vieux Prague, une sensibilité crépusculaire analogue émane de leurs œuvres, suscitant un rapprochement aussi inusité qu'instructif. Cette sensibilité, marquée par les valeurs du dépérissement et de la déperdition, a pourtant entraîné les deux auteurs vers deux directions distinctes : Hofmannsthal vers la révélation des défaillances auxquelles aboutit la revendication individualiste et Leppin vers l'exploration des abîmes ouverts par la haine de soi.

Als Leitfiguren des "Jungen Wien" und des "Jungen Prag" haben Hugo von Hofmannsthal und Paul Leppin in den letzten Jahren des 19. Jahrhunderts eine "Moderne" vertreten, die sich durch ihren Bezug zur Vergangenheit von jener unterscheidet, die sich zur selben Zeit in Berlin entwickelt hat. Hat der Wiener Dichter unablässig seine Verbundenheit mit den romanischen, germanischen und slawischen Kulturen, die alle drei zu seiner Identität gehören, bekräftigt (und verstärkt), so hat der Prager Schriftsteller die beunruhigende Stimmung des alten Prag eingefangen; die Werke beider strahlen aber eine ähnlich dämmerhafte Sensibilität aus und ihre Gegenüberstellung erweist sich als ebenso ungewöhnlich wie aufschlussreich. Diese von Verfall und Verlust geprägte Sensibilität hat die beiden Autoren jedoch in verschiedene Richtungen geführt: Hofmannsthal zur Enthüllung der Schwächen, die der Individualismus nach sich zieht, Leppin zur Erforschung der Abgründe, die der Selbsthass eröffnet.

\section{INDEX}

Mots-clés : Vienne, Prague

\section{AUTEURS}

\section{PATRICK BERGERON}

University of New Brunswick, Fredericton (Canada) 